\title{
Activity Inhibition: A Predictor of Lateralized Brain Function During Stress?
}

\author{
Oliver C. Schultheiss \\ Friedrich-Alexander University
}

\author{
Kathrin Riebel \\ Johannes-Gutenberg University
}

\author{
Nicolette M. Jones \\ New York University
}

\begin{abstract}
The authors tested the hypothesis that activity inhibition (AI), a measure of the frequency of the word "not" in written material, marks a propensity to engage functions of the right hemisphere (RH) and disengage functions of the left hemisphere (LH), particularly during stress. Study 1 and Study 2 showed that high AI predicts faster detection of stimuli presented to the RH, relative to the LH. Study 2 provided evidence that the AI-laterality effect is specific to perceptual, but not motor, laterality and that it is particularly strong in individuals with low mood, but absent in individuals in a positive mood state. Study 3 showed that negative affective stimuli prime the AI-laterality effect more strongly than positive affective stimuli. Findings from Study 4 suggest that situationally induced frustration (losing a contest), in conjunction with high AI, leads to increased attentional laterality. The present findings substantially bolster the construct validity of $\mathrm{AI}$ and contribute to a better understanding of earlier findings linking $\mathrm{AI}$ to physiological stress responses, immune system functioning, alcohol abuse, and nonverbal behavior.
\end{abstract}

Keywords: activity inhibition, laterality, stress, content analysis, self-regulation

Since its conception, activity inhibition (AI), a measure of how frequently individuals use the word "not" in verbal or written language, has been an enigmatic linguistic marker of emotional and motivational differences between individuals. It was originally derived from computerized text analyses of folktales collected from native peoples around the world and found to be associated with population differences in alcohol consumption (McClelland, Davis, Kalin, \& Wanner, 1972). Later on, AI was often routinely scored in imaginative stories written by research participants in laboratory studies in response to picture or verbal cues, a procedure that was primarily aimed at the assessment of individuals' implicit needs for power, affiliation, or achievement (see Schultheiss \& Pang, 2007). And quite frequently, AI would either directly or in interaction with implicit need measures predict outcome variables as diverse as spousal abuse (Mason \& Blanken-

Oliver C. Schultheiss, Institute of Psychology, Friedrich-Alexander University, Erlangen, Germany; Kathrin Riebel, Institute of Psychology, Johannes-Gutenberg University, Mainz, Germany; Nicolette M. Jones, School of Medicine, New York University.

Parts of this research were supported by a National Institutes of Mental Health Grant (R03 MH63069-01/02), a National Science Foundation Grant (BCS-0444301), and by a Horace H. Rackham School of Graduate Studies faculty grant. Kathrin Riebel was supported by a scholarship of the Studienstiftung des deutschen Volkes. We thank Patricia Reuter-Lorenz and Joachim C. Brunstein for their helpful comments on a draft of this article. We are indebted to the following people for helping us conduct this research: Casey Kley (data collection for Study 2), Albert Bertram (synchronizing the computers and data collection in Study 4), Baxter Allen and Steven Stanton (data collection in Study 4).

Correspondence concerning this article should be addressed to Oliver C. Schultheiss, Institute of Psychology, Kochstrasse 4, Friedrich-Alexander University, 91054 Erlangen, Germany. E-mail: oliver.schultheiss@ @sy.phil .uni-erlangen.de ship, 1987), persuasive communication (Schultheiss \& Brunstein, 2002), cardiovascular disease (McClelland, 1979), mood (Langens \& Stucke, 2005), or immune system functioning (McClelland, Davidson, \& Saron, 1985).

Yet despite the success of AI as a predictor of important outcomes, attempts at describing the meaning and function of $\mathrm{AI}$ as a construct have scarcely ventured beyond taking the measure at face value. The label activity inhibition itself reflects the long-held assumption that negations of actions in spoken or written language correspond to the inhibition of emotional impulses and behavior in real life (see McClelland, 1979; McClelland et al., 1972). In our view, however, it is not clear whether this assumption is warranted, particularly in light of research showing that AI is often associated with more, not less, physiological activation (e.g., Fontana, Rosenberg, Marcus, \& Kerns, 1987) and expressive behavior (e.g., Schultheiss \& Brunstein, 2002; see below). More than 40 years after its emergence from computerized text analyses, AI therefore remains a successful measure in need of a good theory.

Our aim in this article is to provide a better understanding of AI as a psychological construct. More specifically, we will make the case that AI represents a propensity to engage functions of the right hemisphere (RH) and disengage functions of the left hemisphere (LH), particularly in response to stressful stimuli and situations. ${ }^{1}$ To support our AI-laterality hypothesis, we will, after a short summary of the measurement credentials of AI, first review the evidence for AI's considerable predictive validity in more

\footnotetext{
${ }^{1}$ When we talk about lateralized hemispheric functions, we do not wish to imply that one entire hemisphere becomes active or the other falls silent. Rather, for the sake of clear writing we use the term hemispheric engagement (or processing) as a shorthand for the idea that certain functions and the specific neural systems supporting them become more strongly engaged in one hemisphere than in the other.
} 
detail and highlight parallels between the correlates of AI on the one hand and correlates of functional hemispheric asymmetry on the other. We will then describe findings from four empirical studies that support the AI-laterality hypothesis.

\section{Measurement Credentials of AI}

Assessment of AI is straightforward: It is a count of how many times a person uses the word "not" in its full or contracted form in oral or written language, often corrected for the total length of the text, with which AI raw scores tend to correlate positively (McClelland, 1979; Pang \& Schultheiss, 2005). AI is most frequently assessed from the Picture Story Exercise (PSE), a method of collecting imaginative stories for the content-coding of motivational needs (Schultheiss \& Pang, 2007), but it can also be coded from any other kind of verbal material, such as folktales, children's readers, or political speeches (e.g., McClelland et al., 1972; Winter, 1991). Coding objectivity is close to perfect (see Langens \& Stucke, 2005; Schultheiss \& Rohde, 2002), and disagreements between coders are typically attributable only to errors of omission. In fact, once text material is transcribed into a computer file, coding objectivity problems can be largely bypassed by using automated coding systems such as the Linguistic Inquiry and Word Count software (LIWC; Pennebaker, Francis, \& Booth, 2001). Internal consistency and test-retest reliability of AI, as assessed from the 5 to 6 picture stories and thus 500 to 600 words typically collected on the PSE, is relatively low, with estimates in the .50 to .60 range (Schultheiss \& Brunstein, 2002). Nevertheless, AI predicts, or is associated with, an impressive array of phenomena, which may explain the peculiar longevity of this atheoretically derived measure.

\section{Parallels Between AI and Hemispheric Asymmetry}

In this section, we will review the major validity correlates of the AI variable and examine to what extent these correlates have also been linked to lateralized brain functions. Specifically, we will review evidence for each of the following validity correlates: negative affect and nonverbal expressiveness, sympathetic activation and cardiovascular disease, immunocompetence and health, and alcohol abuse.

\section{Affect and Nonverbal Behavior}

High levels of AI have been linked to stress-induced negative affect and to nonverbal expressiveness. Langens and Stucke (2005) reported that high levels of AI predict an implicit measure of negative affect cross-sectionally and the experience of daily hassles longitudinally. This suggests that high-AI individuals are more sensitive to stressors than low-AI individuals. McClelland and Kirshnit (1988) found that watching a movie with negative emotional content significantly increased both self-reported negative emotions and AI levels in participants, whereas watching a movie with positive emotional content film did not.

In a study by Schultheiss and Brunstein (2002), the combination of high AI and a strong need for power predicted high levels of nonverbal expressiveness (facial and hand gestures) when participants attempted to convince another person of their point of view on a controversial topic. Nonverbal expressiveness in turn pre- dicted persuasiveness as judged by external observers, a finding that may partly explain why power-motivated individuals with high AI are socially successful (e.g., McClelland \& Boyatzis, 1982; McClelland \& Franz, 1992).

These findings are echoed by the hemispheric laterality literature. Activation of prefrontal, temporal, and parietal regions of the $\mathrm{RH}$ and prefrontal deactivation of the $\mathrm{LH}$ are selectively involved in transient and chronic negative states related to depression and anxiety (Davidson, 2000; Heller, Koven, \& Miller, 2003). The RH is also more strongly involved in nonverbal expression, as evidenced by the more pronounced and unconstrained display of emotions in the left half of the face (controlled by the RH) and the loss of nonverbal expressiveness after RH lesions (Buck \& Duffy, 1980; Dimberg \& Petterson, 2000; Sackeim, Gur, \& Saucy, 1978).

\section{Sympathetic and Cardiovascular Activation}

The relationship between AI and activation of the sympathetic nervous system and the cardiovascular system has frequently been studied from the vantage point of power motivation. McClelland (1979) hypothesized that individuals characterized by high power motivation and high AI (inhibited power motivation, or IPM) are more likely to suppress aggressive impulses, which prevents them from discharging sympathetic arousal and thereby eventually leads to hypertension and cardiovascular disease. In support of this hypothesis, he demonstrated that IPM longitudinally predicted elevated blood pressure and increased incidence of cardiovascular disease and mortality. McClelland and colleagues later solidified and extended the link between IPM and sympathetic activation by showing that IPM in combination with power stress leads to high sympathetic catecholamine levels (McClelland et al., 1985; McClelland, Floor, Davidson, \& Saron, 1980). A subsequent study by Fontana et al. (1987) suggested that the sympathetic-arousal effects observed by McClelland and colleagues are specifically predicted by AI. Examining direct effects of power motivation and AI on blood pressure changes in response to mildly stressful tasks, Fontana et al. (1987) found high levels of AI to be the stronger predictor of blood pressure increases than either power motivation or the IPM pattern.

Psychophysiological research shows that sympathetic modulation of heart rate and blood pressure are more strongly regulated by the RH than the LH. Wittling and colleagues presented emotionally arousing (e.g., stressful or erotic) or neutral movies to the left visual field (LVF) or right visual field (RVF) of their participants (Wittling, 1990, 1997; Wittling, Block, Genzel, \& Schweiger, 1998). They found that emotional movies presented in the LVF, and thus to the $\mathrm{RH}$, elicited much stronger increases in blood pressure and pump capacity of the heart than emotional movies presented to the RVF, and thus to the LH. Similar findings have also been reported by others (see Wittling, 1995, for a review). Moreover, noradrenergic pathways are lateralized to the $\mathrm{RH}$ and are critically involved in arousal, vigilance, and efficient responding to threat (Toga \& Thompson, 2003), suggesting a key role of the RH in sympathetic arousal and control of stress response mechanisms that contrasts with a stronger involvement of the $\mathrm{LH}$ in parasympathetic processes (Hilz et al., 2001; Wittling et al., 1998). 


\section{Immune System Functioning and Health}

High levels of AI have been implicated in impaired immune system functioning and poor physical health. McClelland and Jemmott (1980) identified high AI as a key factor in participants' retrospective reports of severe illnesses. McClelland, Locke, Williams, and Hurst (1982; cited in McClelland, 1987) observed that in men, IPM was associated with low natural killer cell activity and low levels of secretory immunoglobulin A ( $\operatorname{IgA}$ ), representing aspects of the cellular and humoral immune defense, respectively. Finally, McClelland and Kirshnit (1988) observed a significant drop in sIgA in high-AI participants after they had watched a mildly stressful film. This effect did not occur in participants who had watched a nonstressful film.

Research on the involvement of the cerebral hemispheres suggests that there are two ways in which the LH and the RH may be involved in immune system functioning and, ultimately, physical health, one direct, the other indirect. The direct pathway is constituted by the LH's support of immune system functions (particularly cellular immunity, but also humoral immunity; see, for instance, Davidson et al., 2003), a role that is consistent with its parasympathetic, trophotropic functions (Meador et al., 2004; Neveu, 2002). The indirect pathway results from the involvement of the RH not only in sympathetic arousal, but also in the enhancement of the hypothalamic-pituitary-adrenal (HPA) axis response to stress (Wittling, 1995), which leads to the release of the stress hormone cortisol. Cortisol levels are increased under conditions of uncontrollable stress, an effect that can have debilitating effects on the immune system's ability to deal with pathogens (Sapolsky, 1992).

\section{Alcohol Abuse}

As previously mentioned, high AI levels are characteristic of folktales from native tribes that are not prone to abuse alcohol; conversely low AI in such stories is associated with a relatively high level of alcohol abuse (McClelland et al., 1972). Research on U.S. American samples replicated this finding: individuals who frequently consumed hard liquor had lower AI scores on the PSE than less inebriation-prone individuals (Kalin, McClelland, \& Kahn, 1965). McClelland et al. (1972) also observed that AI decreases after the consumption of liquor. Although these studies had cross-sectional or quasi-experimental designs and do not allow to make strong inferences, they do consistently indicate an association between low AI and alcohol (ab)use.

People who abuse alcohol show deficits in RH function (see Ellis \& Oscar-Berman, 1989; Evert \& Oscar-Berman, 2001; Hutner \& Oscar-Berman, 1996). For instance, normal individuals detect and identify emotional stimuli presented more quickly or reliably in the LVF than in the RVF, owing to the RH's specialization on spatial functions and emotional information processing (Borod, 1992; Heilman, 1995). Alcohol abusers, in contrast, show impaired performance on stimuli that are presented to their RH compared with stimuli presented to their LH (Evert \& OscarBerman, 2001; Hutner \& Oscar-Berman, 1996). It is also notable that the characteristic social and behavioral disinhibition associated with alcohol abuse (e.g., Patterson \& Newman, 1993) is more consistent with the approach motivation function ascribed to the $\mathrm{LH}$ than the withdrawal function ascribed to the RH (Davidson, 2000).

\section{Summary}

Our literature review highlights suggestive parallels between lateralized brain functions and AI. Both high AI and RH functions, compared to low AI and LH functions, are associated with higher levels of negative affect, greater nonverbal expressiveness, stronger physiological stress responses, greater cardiovascular activation, less efficient immune system functioning, and less alcohol consumption. Notably, many of the reviewed associations between AI or lateralized brain function on the one hand and outcome variables on the other only become manifest in the presence of a stressor. This suggests that AI reflects a latent disposition to regulate hemispheric function in response to stressors or challenges. Substantial correlations between AI and measures of functional hemispheric asymmetry are therefore more likely to be obtained in the presence of stressors than in their absence.

\section{Overview of the Present Research}

To put the AI-laterality hypothesis to a direct test, we conducted four studies in which we assessed AI with a PSE and brain laterality with a dot-probe task (DPT; Mogg \& Bradley, 1999). On the DPT, a small dot is presented in either the LVF or the RVF of the participant, who indicates per key press which side of the screen the dot appeared on. The response latency difference for dots presented in the LVF versus RVF provides an index of the degree to which the participant's RH or LH was faster at detecting and responding to the stimulus.

We used this task as a measure of the lateralization of attention and expected AI to predict greater attentional sensitivity of the RH. In Study 1, we varied dot probe eccentricity parametrically to determine the optimal distance between stimuli presented in the LVF and RVF. In Study 2, we assessed motor and perceptual asymmetries on the DPT independently to address the question of whether AI is more closely related to hemispheric differences in attention proper, in the preparation of motor responses, or both. We also tested whether negative mood, as a marker of stress, is predictive of the AI-laterality effect. In Study 3, happy and angry facial expressions were presented on each DPT trial to explore the impact of emotionally positive and negative stimuli on the AIlaterality link. Finally, in Study 4, we tested whether the AIlaterality effect is stronger after experimentally induced frustration than after reward.

\section{Study 1: AI and Lateralized Attention in Response to Stimuli of Varying Eccentricity}

In this study, we put the AI-laterality hypothesis to a first test and determined the optimal level of stimulus eccentricity (see Hellige \& Sergent, 1986) for measuring AI-dependent laterality effects.

\section{Method}

Participants. Sixty paid undergraduate and graduate students (30 women; 54 self-identified right-handers), with an average age of 21 years, participated in an experiment advertised as a study on attention and performance. Participants were recruited through fliers posted on campus. Psychology majors were not admitted to the study. 
Design. Eccentricity of probe position (inner, middle, outer) and probe location (left vs. right) were varied in a randomized within-subjects design. AI scores represented the between-subjects factor.

Procedure. After participants had given their informed consent, they completed a PSE and the DPT. All instructions, stimuli, and materials in this and the following studies were presented and all responses recorded using the Experimental Run Time System 3.11 (ERTS, Beringer, 2000) on Dell Pentium personal computers with 14 in. cathode-ray color monitors $(86.6 \mathrm{~Hz}$ vertical retrace) and standard keyboards. For the PSE, participants wrote stories on paper sheets.

Activity inhibition. AI was assessed by having participants write imaginative stories about a set of six PSE pictures, which are described in more detail in Pang and Schultheiss (2005). Stories were later coded for AI by a single scorer. On average, participants wrote $610(S D=122)$ words, containing 4.63 $(S D=3.11)$ AIs summed across all six stories. Cronbach's $\alpha$ for raw AI scores was .48. After attenuating the influence of raw-AI score outliers through a logarithmic transformation, AI scores were positively correlated with word count $(r=.37, p<$ $.005)$. They were adjusted for word count by regression, and AI residuals were $\mathrm{z}$-standardized.

Attentional orienting. Participants were seated with approximately $90 \mathrm{~cm}$ distance to the screen and instructed to press the CTRL key corresponding to the screen location (left or right) the dot appeared in. Each trial on the DPT started with a central fixation cross, presented for $500 \mathrm{~ms}$, followed by a mask pair, ${ }^{2}$ presented either $13.5 \mathrm{~cm}$ apart (midpoint to midpoint; inner position), $18.5 \mathrm{~cm}$ apart (middle position), or $21.25 \mathrm{~cm}$ apart (outer position) and for $66 \mathrm{~ms}$; followed by a single dot $(2.5 \mathrm{~mm} \times 2.5$ $\mathrm{mm})$ in the location of the midpoint of one of the masks. Probes subtended visual angles of $4.3^{\circ}, 5.9^{\circ}$, and $6.7^{\circ}$ from the midline. Response time (RT) registration started with probe onset. The probe disappeared, and the trial was terminated upon a response or after $1000 \mathrm{~ms}$ had elapsed since probe onset. If a participant pressed the wrong key, or responded within $150 \mathrm{~ms}$ after probe onset (premature response), or did not respond within 1,000 ms after probe onset (late response), the response was classified as an error and the trial was repeated at the end of the block until a correct response was registered. Our data set therefore did not contain missing observations. Intertrial intervals varied randomly in $50 \mathrm{~ms}$-steps from $500 \mathrm{~ms}$ to $1,250 \mathrm{~ms}$. The foreground color on all trials was white or color (for dot probes and the mask), the background was black. Participants worked on a total of 24 trials, resulting from a 3 (eccentricity) $\times 2$ (probe location) $\times 4$ (repetition) design. Mean RT across all trials of the DPT was $347 \mathrm{~ms}$ $(S D=44 \mathrm{~ms})$.

Statistical analyses. To accommodate our continuous AI variable and to preserve a maximum of test power, we performed all analyses in this study and the following studies using multiple regression and repeated-measures multiple regression procedures in SYSTAT 10.

\section{Results and Discussion}

We first averaged response times separately for each combination of the factors probe location and eccentricity (see Table 1). Next, we submitted these latency measures to a repeated-measures
Table 1

Mean (SD) Response Latencies (in ms) for Left Dot Probes, Right Dot Probes, and Laterality Index Scores (Right Minus Left) and Correlations Between Activity Inhibition (AI) and Laterality Index Scores For Dot-Probe Presentations on the Inner, Middle, and Outer Screen Position (Study 1)

\begin{tabular}{lcccc}
\hline & $\begin{array}{c}\text { Left dot } \\
\text { probe }\end{array}$ & $\begin{array}{c}\text { Right dot } \\
\text { probe }\end{array}$ & $\begin{array}{c}\text { Laterality } \\
\text { index }\end{array}$ & $r_{\text {AI } \times \text { laterality index }}$ \\
\hline Inner position & $347(53)$ & $350(51)$ & $2.5(34.5)$ & -.06 \\
Middle position & $346(50)$ & $340(52)$ & $-6.3(43.2)$ & $.31^{*}$ \\
Outer position & $356(50)$ & $346(47)$ & $-9.4(30.8)$ & -.04 \\
\hline
\end{tabular}

${ }^{*} p<.05$.

regression analysis with $\mathrm{AI}$ as quantitative between-subjects factor. We found a marginally significant effect of probe eccentricity, $F(2,118)=2.55, M S E=718.74$, partial $\eta^{2}=.04, p=.08$, reflecting the fact that participants were faster at detecting dot probes shown in the middle position $(M=343 \mathrm{~ms}, S D=47 \mathrm{~ms})$ than in either the inner $(M=348 \mathrm{~ms}, S D=49 \mathrm{~ms})$ or the outer position $(M=351 \mathrm{~ms}, S D=46 \mathrm{~ms})$. The difference between the averaged RTs on inner and outer positions and the RTs on the middle position was significant, $t(59)=1.96, p=.05$. These findings suggest that a stimulus eccentricity of about $5.9^{\circ}$ from the midpoint of the visual field hits a sweet spot for detection by the hemisphere contralateral to the side of presentation, possibly because stimuli are presented extrafoveally enough to prevent detection by and thus response competition from the ipsilateral hemisphere, but not yet too peripherally to hinder quick detection.

The AI $\times$ probe location $\times$ eccentricity effect also became significant, $F(2,116)=4.33, M S E=500.16$, partial $\eta^{2}=.07, p<$ .05 . To follow up on this finding, we created laterality index scores by subtracting averaged left probe RTs from averaged right probe RTs separately for inner, middle, and outer position trials. Higher laterality scores thus reflect relatively greater RH sensitivity. As shown in Table 1, AI was associated with greater relative $\mathrm{RH}$ responsiveness on trials in which the probe was shown in the middle positions, but not on trials in which the probe was presented either in the inner or outer left and right positions. Thus, despite the relatively small number of DPT trials on which our laterality index was based, this study provided initial support for the AI-laterality hypothesis.

\section{Study 2: Differential Association Between AI and Perceptual and Motor Laterality}

Although Study 1 helped us determine the optimal stimulus eccentricity on the DPT for the assessment of AI-associated laterality, it did not allow us to draw conclusions about whether AI in fact predicted lateralized attentional orienting (a perceptual process), or, alternatively, a disposition of the RH to initiate motor responses to a stimulus faster than the $\mathrm{LH}$, or a combination of

\footnotetext{
${ }^{2}$ The mask pair was included to prepare participants for a later portion of the DPT unrelated to the present findings. We do not think that the inclusion of the mask pair has had a substantial impact on the results reported for this study (see also Study 2).
} 
both. In Study 2, we therefore aimed at disentangling perceptual and motor laterality by varying response hand independently of stimulus laterality (see Saron, Foxe, Schroeder, \& Vaughan, 2003). Because we had hypothesized that AI has a stronger effect on behavior if a person is stressed (e.g., McClelland et al., 1985), we included a mood measure as a marker of participants' current stress levels and expected the AI-laterality effect to be stronger in participants who were in a negative mood than in participants who were in a positive mood.

\section{Method}

Participants. Seventy-eight undergraduate students (32 women; 68 self-identified right-handers) with an average age of 19 years participated for course credit in an experiment advertised as a study on attention and performance.

Design. Probe location (left vs. right) and response hand (left vs. right) were varied in a randomized within-subjects design. AI scores represented the between-subjects factor.

Procedure. Up to 6 participants were scheduled and tested in single sessions. After participants had given their informed consent, they completed a mood measure, a PSE, and the DPT.

Activity inhibition. The same PSE and procedures as in Study 1 were used to assess AI. On average, participants wrote 536 $(S D=116)$ words, containing $3.94(S D=2.57)$ AIs summed across all six stories. Cronbach's $\alpha$ for raw AI scores was at .55. Because AI scores were not substantially correlated with word count $(r=.05, p>.10)$, we did not correct them for protocol length in this study.

Attentional orienting. The DPT followed the same procedures as described in Study 1 with the following exceptions: First, left and right dot presentation positions were always $18.5 \mathrm{~cm}$ apart on the screen (i.e., $5.9^{\circ}$ visual angle from the midpoint of the screen). Second, after an initial warm-up period, all participants were instructed to respond only with their left hand on the first and only with their right hand on the second block of DPT trials. Probe location was randomized within blocks. On the left-hand block, participants responded to LVF dot presentations by pressing the left standard-keyboard arrow key with their middle finger and to RVF dot presentations by pressing the right arrow key with the left index finger. On the right-hand block, they used the right index finger to respond to LVF dot presentations and the right middle finger to respond to RVF dot presentations. Third, no mask was presented between fixation cross and dot probe. Because trials with late or incorrect responses were repeated until correctly solved, our data set did not contain missing data. Participants worked on a total of 64 trials, resulting from a 2 (probe location) $\times 2$ (response hand) design, with 16 within-block realizations of the probe location factor. Mean RT across all DPT trials was $326 \mathrm{~ms}$ ( $S D=$ $36 \mathrm{~ms}$ ).

Self-reported mood. Mood was assessed with the hedonic tone scale from the University of Wales Mood Adjective Check List (Matthews, Jones, \& Chamberlain, 1990), consisting of the items happy, satisfied, contented, cheerful, sad, depressed, dissatisfied, and sorry. Items were presented in random order with the primer "Right now I feel ..." and participants could endorse each item on a 4-point scale featuring the gradations definitely not (1), slightly not (2), slightly (3), and definitely (4). After recoding of negative affect items sum scores were calculated for overall hedonic tone $(M=23.18, S D=5.14)$. Internal consistency (Cronbach's $\alpha)$ for the hedonic tone-scale was .83 .

\section{Results and Discussion}

We first averaged response times separately for each combination of the factors probe location and response hand, resulting in the following mean $(S D)$ response latency scores (in $\mathrm{ms}$ ): left hand/LVF, 323 (37); left hand/RVF, 325 (39); right hand/LVF, 333 (40); right hand/RVF, 322 (40). Next, we submitted these latency measures to a repeated-measures regression analysis with AI as quantitative between-subjects factor. The probe location $\times$ response hand interaction was significant, $F(1,77)=21.50$, $M S E=162.14$, partial $\eta^{2}=.22, p<.00001$, indicating faster responses on uncrossed trials (stimulus and response are processed by the same hemisphere) than on crossed trials (stimulus and response are processed by different hemispheres), a finding that is consistent with the literature on interhemispheric information transfer (see Saron et al., 2003). The AI main effect and the probe location $\times \mathrm{AI}$ interaction effect were significant, $F(1,76)=6.08$, $M S E=4833.51$, partial $\eta^{2}=.07, p=.01$, and $F(1,76)=3.75$, $M S E=260.24$, partial $\eta^{2}=.05, p=.05$, respectively. But neither the response hand $\times$ AI effect nor the probe location $\times$ response hand $\times \mathrm{AI}$ effect reached significance, suggesting that $\mathrm{AI}$, or the association between AI and perceptual laterality, does not depend on whether the motor response is driven preferentially by the $\mathrm{LH}$ or the RH.

Participants' AI scores were positively correlated with their average response latencies, $r=.27, p=.01$, indicating that higher levels of AI were associated with overall slower responding to dot probes. To follow up on the significant probe location $\times$ AI interaction, we created perceptual laterality scores, collapsing across response hand, according to the formula: (left hand/RVF + right hand/RVF) $\div 2$ minus (left hand/LVF + right hand/ LVF $) \div 2$. Perceptual laterality scores $(M=-4.2, S D=16.4)$ were significantly correlated with $\mathrm{AI}(r=.22, p=.05)$, indicating that high levels of AI were associated with a tendency to more quickly detect stimuli presented to the RH than to stimuli presented to the LH, regardless of which hemisphere was in charge of responding to the stimulus.

To explore the influence of mood on the AI-laterality association, we next ran a multiple regression equation with the perceptual laterality index as dependent variable and AI, hedonic tone, and the AI $\times$ Hedonic Tone interaction term as predictors. While mood did not have a significant main effect on perceptual laterality, the interaction term was significant, $B=-3.30, S E=1.35$, semipartial $\mathrm{r}=-.27, t(74)=-2.44, p=.02$. In participants with mood scores below the median, high AI scores were significantly associated with a strong advantage for detecting stimuli presented to the $\mathrm{RH}, r(38)=.43, p<.01$, whereas the correlation between $\mathrm{AI}$ and perceptual laterality was nonsignificant in participants with mood scores at or above the median, $r(40)=.01, n s$ (see Figure 1).

Thus, the results of this study indicate that high AI is specifically associated with slower detection of stimuli presented to the $\mathrm{LH}$ than stimuli presented to the $\mathrm{RH}$ and that the AI-laterality link is particularly strong in the context of negative mood, but absent in the context of positive mood. Our findings also suggest that AI is not associated with the lateralization of simple motor responses. 

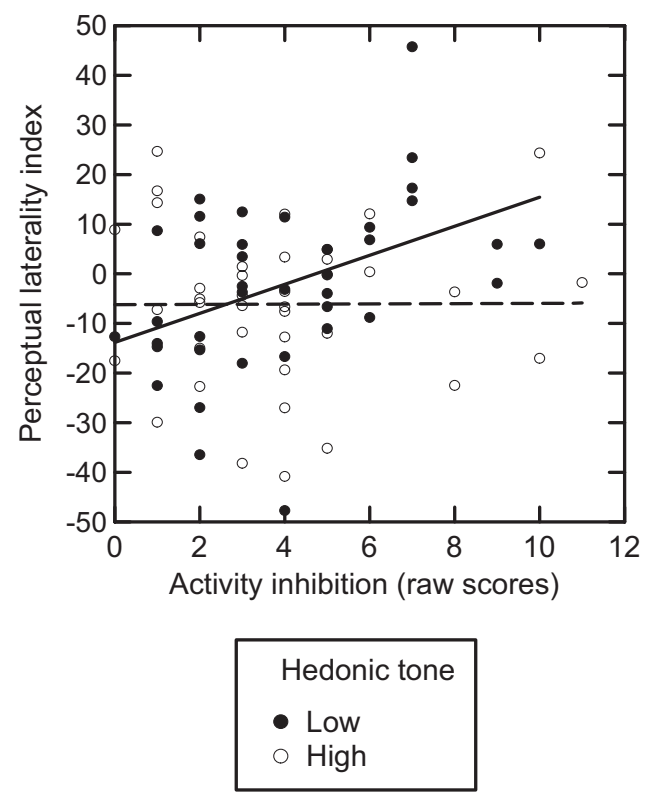

Figure 1. Activity Inhibition $\times$ Hedonic Tone (median-split) interaction on perceptual laterality (positive scores $=$ faster responses to stimuli presented to the right hemisphere) in Study 2. High hedonic tone: striped line; low hedonic tone: solid line.

But given the limitations of our adaptation of the DPT in this study (relatively few trials, no counterbalancing of response hand sequence), this conclusion needs further corroboration.

\section{Study 3: AI and Lateralized Attention on an Emotional DPT}

To study whether affective state has a causal effect on AIdependent laterality, we reanalyzed data from a study of the effects of motivational needs on attentional orienting to emotional faces (Schultheiss \& Hale, 2007; Study 2). In this study, participants were shown pairs of identical faces, one displaying an emotional expression, the other displaying a neutral expression, before each dot-probe presentation. Although Schultheiss and Hale used angry, happy, and surprised faces in their study, we focused only on the clearly valenced anger and joy face trials to examine priming effects of negative and positive emotional stimuli on the AIlaterality association. Based on previous research suggesting that emotional face processing engages RH functions (Adolphs, 2002; Borod, 1992), particularly in the case of negative emotional expressions (Reuter-Lorenz \& Davidson, 1981), we expected joy face trials and particularly anger face trials to elicit faster responses in high-AI individuals compared with low-AI individuals.

\section{Method}

Participants. Sixty paid undergraduate and graduate students (31 women; 58 self-identified right-handers) with an average age of 20 years participated in an experiment advertised as a study on attention and performance. Participants were recruited through fliers posted on campus. Psychology majors were not admitted to the study.
Design. Emotion (joy, anger), emotional face location (left vs. right), probe location (left vs. right), and face exposure duration (12 ms, $116 \mathrm{~ms}, 231 \mathrm{~ms}$ ) were varied in a within-subjects design, with the first three factors varied randomly within exposure duration. Exposure duration sequence (ascending, descending) was balanced across participants. AI scores represented the betweensubjects factor.

Procedure. Participants were scheduled and tested in groups of up to four. After participants had given their informed consent, they completed a PSE, the DPT, and various other tasks.

Activity inhibition. The same PSE and procedures as in Study 1 were used to assess AI. On average, participants wrote 642 $(S D=128)$ words, containing $4.90(S D=3.10)$ AIs summed across all six stories. Cronbach's $\alpha$ for raw AI scores was .37. AI scores were positively correlated with word count $(r=.42, p<$ .001). They were adjusted for word count by regression, and AI residuals were $z$-standardized.

Stimulus materials. For the DPT we used digitized slides of all Caucasian and Japanese posers (two exemplars for each race/ gender/emotion combination) displaying joy and anger from Matsumoto and Ekman (1988). Faces were cropped so that each was visible from cheekbone to cheekbone and hairline to chin, and picture portions below the jawline were blackened. Faces were resized to $12.0 \mathrm{~cm}$ height after cropping (width could vary, depending on posers' physiognomy). A $12.2 \mathrm{~cm}$ (height) $\times 9.0 \mathrm{~cm}$ (width) mask was created by copying fragments from each poser's neutral expression onto a black background such that the combined fragments resembled the contours and proportions of a regular face, but did not show any feature of the face (e.g., an eye, mouth, nose) as a whole.

Attentional orienting. Each trial on the DPT started with a central fixation cross, presented for $500 \mathrm{~ms}$, followed by a face pair, presented $18.5 \mathrm{~cm}$ apart (midpoint to midpoint) for either 12 $\mathrm{ms}, 116 \mathrm{~ms}$, or $231 \mathrm{~ms}$; followed by a mask pair, presented 18.5 $\mathrm{cm}$ apart (midpoint to midpoint; $9.5 \mathrm{~cm}$ distance between inner edges) and for $66 \mathrm{~ms}$, in place of the previous face pair; followed by a single dot in the location of the midpoint of one of the faces. The DPT was identical in all other regards with the one described in Study 1. Analyses are based on a total of 192 trials, resulting from a 2 (emotion: joy vs. anger) $\times 2$ (face gender: male vs. female $) \times 2$ (emotional face location: left vs. right) $\times 2$ (probe location: left vs. right) $\times 2$ (face exemplars: first vs. second) $\times 2$ (face ethnicity: Japanese vs. Caucasian) $\times 3$ (face exposure duration: 12, 116, $231 \mathrm{~ms})$ factorial. Mean RT was $374 \mathrm{~ms}(S D=$ $39 \mathrm{~ms})$.

\section{Results and Discussion}

We subjected participants' response latencies, averaged across male and female Japanese and Caucasian faces, to a repeatedmeasures regression analysis with emotion (anger, joy), emotional face location (left, right), dot probe location (left, right), and exposure duration (12 ms, $116 \mathrm{~ms}, 231 \mathrm{~ms})$ as within-subjects factors and participants' AI scores as a quantitative betweensubjects factor. We found a significant effect for the AI $\times$ probe location term, $F(1,58)=7.44, M S E=6,021.55$, partial $\eta^{2}=.11$, $p<.01$, and a marginally significant AI $\times$ Probe Location $\times$ Emotion effect, $F(1,58)=2.73, M S E=1,115.93$, partial $\eta^{2}=$ $.05, p=.10$, suggesting that the effect of $\mathrm{AI}$ on response laterality 
was modulated by the type of emotion shown. Notably, the AI $\times$ probe location $\times$ emotion location and the $\mathrm{AI} \times$ probe location $\times$ emotion location $\times$ emotion interactions were not significant, indicating that the relationship between $\mathrm{AI}$ and responding to lateralized probes did not depend on whether an emotional face had been shown on the same side of the screen on which the probe appeared or on the other side. To follow up on the interaction effects, we created global laterality index scores by subtracting averaged responses to probes presented in the LVF from averaged response times to probes presented in the RVF, collapsing across all other factors. We also created laterality index scores separately for trials on which anger faces were shown and trials on which joy faces were shown. As shown in Table 2 and Figure 2, high-AI participants, relative to low-AI participants, were faster to detect dot probes presented in their LVF than to dot probes presented in their RVF. Moreover, this difference was more pronounced in response to anger-face trials than to joy-face trials. The former effect supports the notion that high AI is associated with greater $\mathrm{RH}$ than LH activation in the presence of negative affective stimuli. The latter effect, although weak, is consistent with past research suggesting that the $\mathrm{RH}$ is involved in the processing not only of negative, but also of positive facial expressions (Adolphs, 2002; Borod, 1992; Maxwell, Shackman, \& Davidson, 2005).

\section{Study 4: AI and Lateralized Attention After Situationally Induced Success or Frustration}

In this study, we tested whether situationally induced frustration produces increases in the AI-laterality effect. For this purpose, we analyzed data collected in a study on the effects of power motivation and dominance contest outcome (winning vs. losing) on cortisol changes reported by Wirth, Welsh, and Schultheiss (2006).

\section{Method}

Participants. One hundred sixteen undergraduate and graduate students of the University of Michigan (mean age $=20.3$ years) participated in this study in same-sex pairs for payment. Psychology majors were not admitted to the study. Seven participants were discarded from analysis, because they either had missing data due to technical problems or had recently participated in a similar study with false contest feedback. Thus, data from a total of 109 participants (53 women and 56 men; 99 self-identified right-handers) were used in this study.

Design. Participants were randomly assigned to the winning and losing conditions constituting the factor Contest Outcome. Fifty-five students participated in the lose condition, 54 in the win

Table 2

Correlations Between Laterality Index Scores (Right Minus Left) and Activity Inhibition Scores (Study 3)

\begin{tabular}{llll}
\hline & $r$ & $M$ & $S D$ \\
\hline Overall & $.34^{* * *}$ & 3.0 & 23.6 \\
Anger & $.42^{* * * *}$ & 5.7 & 23.6 \\
Joy & $.22^{\dagger}$ & 0.2 & 27.3
\end{tabular}

Note. Positive scores/correlations signify right-hemispheric bias. ${ }^{\dagger} p<.10 .{ }^{* * *} p<.01 .^{* * * * *} p<.001$.
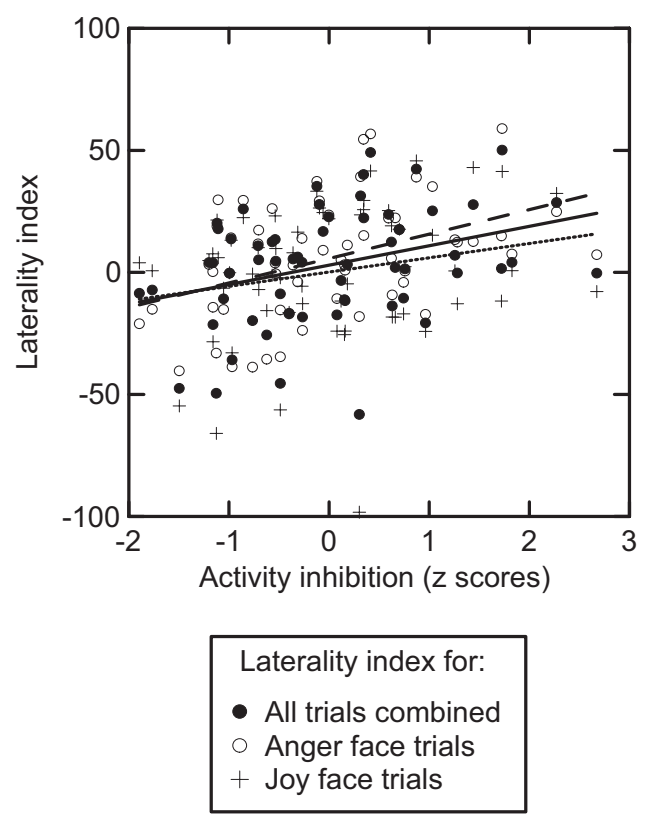

Figure 2. Effect of activity inhibition on perceptual laterality (positive scores $=$ faster responses to stimuli presented to the right hemisphere) as a function of face stimulus type (anger [striped line], joy [dotted line], combined [solid line]) in Study 3.

condition. Individual differences in AI and lateralized attention were assessed at baseline and after the contest and were used as quantitative predictors or dependent variables in the analyses. Changes in hedonic tone from baseline to after the contest were assessed to check for the effect of contest outcome on participants' affective state.

Procedure. Sessions were run by a single male or female experimenter. As part of hypotheses unrelated to those tested here, participants were administered, in a double-blind fashion, $200 \mathrm{mg}$ caffeine or placebo (vitamin C) at the beginning of the study. In the precontest phase, participants completed a mood questionnaire, a PSE, a DPT and other tasks. Next, the experimenter announced that participants would compete against each other in a contest based on a speed-based task.

During the contest phase, participants competed against each other on 10 rounds of a serial-response task, with a total duration of $10 \mathrm{~min}$ and bogus performance feedback after each round. Details on this task, which was designed to be strongly rewarding (victory) or frustrating (defeat), are given in Schultheiss et al. (2005). Participants in the winning condition "won" all rounds except for the second and the fifth, and participants in the losing condition correspondingly "lost" all rounds except for the second and the fifth.

In the postcontest phase, participants first worked on another mood questionnaire, and then completed a second PSE and DPT. Finally, they completed a background-data questionnaire, a suspicion check and were fully debriefed. None of the 109 participants demonstrated awareness that the contest outcome was rigged.

Activity inhibition. A detailed description of the baseline PSE used in this study is given in Wirth et al. (2006). In addition to the five pictures described by these authors, we also used AI counts 
from a sixth picture, showing a woman giving a presentation and used for the first time in this study. For the postcontest PSE, we used the pictures hooligan attack, woman and man arguing, and ice hockey duel. All three pictures were used for the first time in this study. The same scoring procedures as in Study 1 were used to assess AI. On the baseline PSE, participants wrote 633 (SD = $137)$ words, containing $5.61(S D=3.75)$ AIs summed across all six stories. On the postcontest PSE, participants wrote $316(S D=$ $75)$ words, containing $2.60(S D=2.33)$ AIs summed across all three stories. Cronbach's $\alpha$ for raw AI scores was at .57 for the baseline PSE and .50 for the postcontest PSE. AI scores were positively correlated with word count on both the baseline PSE $(r=.45, p<.000001)$ and the postcontest PSE $(r=.46, p<$ .000001). They were adjusted for word count by regression, and AI residuals were $z$-standardized.

Attentional orienting. The same DPT procedures as in Study 1 were used, with the following exceptions: First, participants used a 4-key keypad for response registration, with one key assigned for left-dot/left-hand responses and one for right-dot/right-hand responses. Second, left and right dot presentation positions were always $18.5 \mathrm{~cm}$ apart on the screen (i.e., $5.9^{\circ}$ visual angle from the midpoint of the screen). On both the baseline and postcontest DPT, participants worked on a total of 24 trials, resulting from a 2 (probe location) $\times 12$ (repetition) design. Mean RT across all trials was $323 \mathrm{~ms}(S D=49 \mathrm{~ms})$ on the baseline DPT and $308 \mathrm{~ms}(S D=49$ $\mathrm{ms}$ ) on the postcontest DPT.

Self-reported mood. The same hedonic tone scale as in Study 2 was administered. Cronbach's $\alpha$ for the hedonic tone scales was .82 at baseline and .86 postcontest.

\section{Results and Discussion}

We first tested whether our manipulation had worked by examining the effect of contest outcome on participants' affective state. Relative to their baseline hedonic tone levels $(M=25.22)$, losers had significantly lower mood after the contest $(M=21.07)$, $t(54)=-8.19, p<.00000005, d=-2.23$. In contrast, winners registered a significant increase in their hedonic tone levels from $M=25.57$ at session start to $M=27.26$ after the contest, $t(52)=3.78, p<.0005, d=1.05$. The Time $\times$ Contest outcome interaction was significant, $F(1,106)=73.68, M S E=6.34$, partial $\eta^{2}=.41, p<.000001$. Thus, experimentally induced defeat was extremely effective in frustrating participants, whereas experimentally induced victory enhanced participants' mood.

After creating precontest $(M=4.59, S D=29.38)$ and postcontest $(M=2.49, S D=31.29)$ laterality indices from participants' DPT RTs as previously described, we examined correlations within and between pre- and postcontest $\mathrm{AI}$ and laterality index measures. As shown in Table 3, both measures had moderate test-retest stability, but we found no significant between-measures correlations before or after the contest. ${ }^{3}$

To test for AI-driven changes in brain laterality after losing versus winning the contest, we next ran a multiple regression analysis with the postcontest laterality index as dependent variable, precontest laterality index as covariate, and precontest AI, contest outcome and the $\mathrm{AI} \times$ Contest Outcome interaction term as predictors. The interaction was significant, $B=-17.63$, $S E=5.90$, semipartial $r=.27, t(104)=-2.99, p<.005,{ }^{4}$ and due to the fact that in losers high levels of AI were strongly
Table 3

Correlations Between Baseline (T1) and Postcontest (T2)

Laterality Index Scores (Right Minus Left) and Activity Inhibition Scores (Residualized z Scores)

\begin{tabular}{lccc}
\hline & 1 & 2 & 3 \\
\hline 1. Laterality index T1 & $-6^{* * *}$ & & \\
2. Laterality index T2 & -.10 & .02 & - \\
3. Activity inhibition T1 & -.09 & .05 & $.30^{* * * *}$ \\
4. Activity inhibition T2 & &
\end{tabular}

Note. In Study 4, positive scores/correlations signify right-hemispheric bias. $N=107$ for all correlations involving T2 measures.

${ }^{* * *} p<.01 .{ }^{* * * *} p<.005$.

associated with rightward shifts in laterality index scores, partial $r=.38, p<.005$, whereas in winners AI scores were not significantly related to changes in the laterality index, partial $r=$ $-.17, p>.10$ (see Figure 3 ). When we repeated the analysis excluding an outlier (a case with a laterality score residual less than -100 ) and five cases with high leverage (AI scores $>2$; see Figure 3 ), the AI $\times$ Contest Outcome interaction remained significant, $B=-22.32, S E=7.29$, semipartial $r=.28, t(104)=$ $-3.06, p<.005$, (partial correlations: .32 for losers and -.27 for winners, $p \mathrm{~s} \leq .05$ ). These findings are consistent with the notion that situationally induced stress and frustration enhances $\mathrm{RH}$ functions and impairs LH functions in high-AI individuals, whereas the AI-laterality association does not manifest itself in rewarding and emotionally pleasing situations.

We also explored whether postcontest changes in AI could be predicted from precontest laterality index scores by running a multiple regression analysis with the postcontest AI scores as dependent variable, precontest $\mathrm{AI}$ as covariate, and precontest laterality index, contest outcome and the Laterality Index $\times$ Contest Outcome interaction term as predictors. The interaction term did not reach significance, $F<1$, suggesting that although shifts

\footnotetext{
${ }^{3}$ Using only the positive affect items of our hedonic tone scale, we also tested whether affect at baseline moderated the effect of AI on the baseline laterality index in participants in the placebo condition. After removal of an outlier (Studentized residual $=-3.9$ ), the AI $\times$ Positive Affect effect was marginally significant, $B=-3.66, S E=1.89, t(47)=-1.94, p=.06$, $d=-0.57$, reflecting the fact that $\mathrm{AI}$ and the laterality index were positively associated among low-positive affect participants $(r=.14, n s)$ and negatively associated among high-positive affect participants $(r=$ $-.07, n s)$. These findings, while not as strong as those obtained in Study 2 and emerging only for the positive affect scale, support our earlier conclusion that mood states as markers of the experience of stress moderate the AI-laterality link. Notably, we found the reverse effect among participants treated with caffeine: here, AI and the laterality index were negatively associated among low-positive affect participants $(r=-.27, n s)$ and largely independent of each other among high-positive affect participants $(r=.02, n s)$; for the AI $\times$ Positive Affect interaction: $B=5.14$, $S E=2.43, t(52)=2.11, p=.04, d=0.59$. The 3-way interaction between AI, pharmacological treatment, and positive affect was significant, $B=8.80, S E=3.06, t(99)=2.87, p<.005, d=0.58$. Thus, the caffeine treatment employed in this study appears to have counteracted the detection of the predicted AI-laterality link in low-mood participants at baseline.

${ }^{4}$ The 3-way interaction of pharmacological treatment, contest outcome, and $\mathrm{AI}$ on the postcontest laterality index was not significant, $p=.86$.
} 


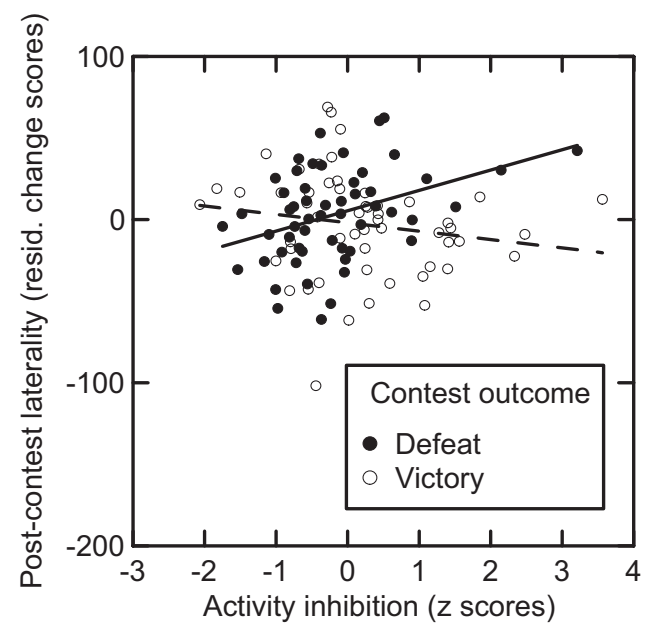

Figure 3. Activity Inhibition $\times$ Contest Outcome interaction on postcontest perceptual laterality scores (residualized for precontest laterality scores; positive scores reflect a rightward shift, negative a leftward shift) in Study 3. Defeat: solid line; victory: striped line.

in lateralized brain function following a frustrating event can be predicted from a person's trait AI level, shifts in AI cannot be predicted from the person's initial laterality index.

\section{Meta-analysis of Findings}

To evaluate the robustness of the AI-laterality effect, we conducted a meta-analysis based on primary data from all 4 studies. AI scores, laterality index scores, and LVF and RVF RT scores were converted to $\mathrm{z}$ scores within each study before pooling the data. Across all 4 studies, we found a reliable association between AI and the laterality index (averaged anger and joy face trials in Study 3, baseline measures in Study 4), $r(307)=.15, p=.01$. When we excluded participants from Study 4 who had taken caffeine, which reversed the AI-laterality effect (see Footnote 3), the association became stronger, $r(251)=.21, p<.001$. Thus, high levels of AI are associated with a greater propensity toward right-lateralized processing of stimuli. We also checked whether the AI-laterality effect was attributable to slower processing in the $\mathrm{LH}$, faster processing in the $\mathrm{RH}$, or both by regressing responses to RVF and LVF stimuli simultaneously on AI. In the full sample, responses to both RVF and LVF stimuli were uniquely associated with AI, $B=0.313, S E=0.097$, semipartial $r=.18$, $t(304)=3.21, p<.005$ and $B=-0.192, S E=0.097$, semipartial $r=-.11, t(304)=-1.97, p<.05$, respectively. After exclusion of caffeine-treated participants from Study 4, the unique associations between RVF and LVF stimulus responses and AI were $B=0.445, S E=0.108$, semipartial $r=.25, t(248)=4.13, p<$ .00005 and $B=-0.276, S E=0.106$, semipartial $\mathrm{r}=-.16$, $t(248)=-2.61, p<.01$, respectively. Thus, across studies, high levels of AI were associated with both slower responses to stimuli presented to the $\mathrm{LH}$ and faster responses to stimuli presented to the RH.

We then examined whether the AI-laterality effect is stronger under conditions of negative mood (below-median hedonic tone in Study 2), threatening stimuli (anger face trials in Study 3), or experimentally induced frustration (losing in Study 4; postcontest laterality scores were adjusted for baseline laterality scores). We found this to be the case, and very reliably so, $r(157)=.38, p<$ .000001. Unique associations between RVF and LVF stimulus responses and AI were $B=0.709, S E=0.141$, semipartial $r=$ $.38, t(154)=5.04, p<.000005$ and $B=-0.538, S E=0.144$, semipartial $\mathrm{r}=-.28, t(154)=-3.75, p<.0005$, respectively (in Study 4, postcontest RVF and LVF latencies were adjusted for precontest latencies). ${ }^{5}$ Conversely, the AI-laterality effect was virtually absent across studies under conditions of positive mood (above-median hedonic tone in Study 2), friendly stimuli (joy face trials in Study 3), or experimentally induced satisfaction (winning in Study 4; adjusted postcontest laterality scores), $r(154)=.02$, $p=.84$. Here, unique associations between RVF and LVF stimulus responses and AI were $B=0.126, S E=0.156$, semipartial $r=.07, t(147)=0.81, p=.42$ and $B=-0.077, S E=0.146$, semipartial $\mathrm{r}=-.04, t(147)=-0.52, p=.60$, respectively.

Twenty-eight left-handers, 280 right-handers, 161 men, and 146 women participated in all studies. In the meta-analysis, Pearson correlations of handedness and gender with AI scores, laterality index scores, and LVF and RVF RT scores were nonsignificant, $p s>.10$. We also tested whether handedness or gender moderated any of the other effects reported in the meta-analysis, but without significant results, $p \mathrm{~s}>.10$.

\section{General Discussion}

Results of all four studies support the hypothesis that high levels of $\mathrm{AI}$ are associated with a propensity toward facilitated $\mathrm{RH}$ functions and impaired LH functions, particularly in response to stressors. Results from Studies 1 and 2 indicate that high AI predicts increased lateralization of attention, as indexed by faster detection of stimuli presented to the $\mathrm{RH}$ than of stimuli presented to the LH. In addition, Studies 2 through 4 provide evidence that the AI-laterality association is greater under conditions of impaired mood (Study 2), the presence of threatening stimuli (anger faces in Study 3), and situationally induced frustration (Study 4).

A meta-analysis of these findings corroborated that (a) AI is reliably associated with right-lateralized attentional sensitivity in general, that is, when stress is not taken into account and (b) the AI-laterality effect is particularly strong when individuals are threatened, frustrated, or are in a negative mood, but not when these conditions are not met. In addition, our meta-analytic findings indicate that high levels of AI are uniquely associated with

\footnotetext{
${ }^{5} \mathrm{We}$ also tested whether the unique associations between AI and responses to LVF and RVF stimuli we obtained in these regressions significantly differed from each other in magnitude. We followed a procedure described by Cohen, Cohen, West, and Aiken (2003) to test for differences in partial Bs from the same sample, which derives a $t$ statistic from dividing the difference between two partial B weights by the standard error of their difference (Appendix 2, formulas A2.9 and A2.10). Because we were interested in differences in effect magnitude, not direction, we multiplied the negative predictor in each regression with -1 so that all Bs became positive. In each case that we tested (full sample; full sample minus caffeine-treated participants; participants tested under conditions of negative mood/threat/frustration), the magnitudes of the $\mathrm{AI} / \mathrm{LH}$ and the $\mathrm{AI} / \mathrm{RH}$ associations did not differ significantly, $t \mathrm{~s}<1.21, p \mathrm{~s}>.05$. Thus, the AI-laterality effect was attributable in equal amount to decreased attentional functions of the $\mathrm{LH}$ and increased attentional functions of the $\mathrm{RH}$.
} 
both faster stimulus detection by the RH and slower detection by the LH. This suggests that AI represents a disposition to dampen $\mathrm{LH}$ functions and facilitate $\mathrm{RH}$ functions.

What kind of picture emerges for the AI variable when our present findings are combined with past research on AI? We suggest that in response to stimuli and events that are associated with punishment (e.g., an angry face) or nonreward (e.g., suffering a defeat in a dominance contest), high levels of AI predispose individuals to engage $\mathrm{RH}$ functions destined to deal with the stressor, as suggested by our finding of quicker detection of dot probes presented to the $\mathrm{RH}$ and by earlier findings linking AI to enhanced physiological stress responses (e.g., McClelland et al., 1985 , 1980), and to disengage from LH functions that are nonessential for countering the stressor, as suggested by our finding of slower detection of dot probes presented to the LH and by earlier findings linking AI to impaired immune system functions (e.g., McClelland \& Jemmott, 1980).

Given the amount of research that has been conducted on hemispheric differences, emotion and personality over the past decades (for overviews, see Davidson \& Hugdahl, 1995; Hugdahl \& Davidson, 2003), one may wonder how our explanation of the AI variable fits into existing models of functional brain asymmetry. We see similarities between effects of AI on laterality with accounts given by Heller et al. (2003) and by Goldberg (2001). Heller and colleagues have presented a model that differentiates between an association between left and right frontal activation and emotional valence (see also Davidson, 2000) and anterior and posterior activation and emotional arousal. Specifically, they argue that anxious arousal is associated with activation in right posterior areas, which support attentional vigilance and orienting (see Posner, 1995; Wittling, 1995), and deactivation in left prefrontal cortex, an effect that is characteristic of impaired mood (see Davidson, 2000). According to Heller and colleagues, right occipitoparietal activation supports attention to stimuli presented in the LVF, which, either independently or combined with RVF-field attentional impairments associated with left-frontal deactivation, gives rise to marked attentional laterality patterns similar to those we observed in high-AI individuals in the present research. In contrast, depressive affect is associated with decreased activation in both left frontal and right posterior regions of the brain, resulting in an absence of marked attentional laterality effects, because responses to stimuli presented to either hemisphere are slowed down about equally (Keller et al., 2000).

We speculate that high AI levels predispose individuals to the engagement of brain systems that Heller et al. (2003) identify with anxious arousal not only because the observed attentional bias for LVF stimuli in high-AI individuals would be consistent with such an account, but also, and perhaps even more importantly, because AI does not seem to predispose individuals to the lack of (adaptive) behavior typically associated with states of depression. Rather, combined with high power motivation, high levels of AI have been found to predict effective nonverbal behavior (e.g., Schultheiss \& Brunstein, 2002) and successful mastery of complex social tasks (e.g., McClelland \& Boyatzis, 1982). We suspect that AI-induced anxious arousal may facilitate the careful assessment of challenging or difficult situations (a function similar to Gray's, 1987, conceptualization of a conflict-resolving behavioral inhibition system) and as a consequence promote flexible behavioral adjustment.
This account would also be consistent with Goldberg's (2001) model of hemispheric differences, which assigns a critical role to the RH for developing effective strategies for dealing with novel challenges from the environment and that require flexible switching from one type of responding to another (see Aron, Robbins, \& Poldrack, 2004; Hugdahl, 1995, for related arguments). Once such strategies are in place, they are stored as routines in the LH. To the extent that $\mathrm{AI}$ is associated with the novelty/routinization difference Goldberg proposed for the two hemispheres, high levels of AI may promote the acquisition of new ways of dealing with the environment, especially when the individual is challenged with signals of punishment or nonreward, whereas low levels of AI may predispose individuals to fall back on established LH-based behavioral routines during stress. We believe that this prediction is particularly worthy of further investigation.

At this point, it may also be important to emphasize what the AI measure is not. AI has little direct overlap with self-report measures of mood states. In Studies 2 and 4, correlations between AI and the hedonic tone scale, our state measure of mood, were -.07 and .13, respectively, and not significant. So far, Langens and Stucke (2005) were the only ones to report a direct relationship between $\mathrm{AI}$ and affect. It is important to point out, though, that in their first study, they obtained this result only with an implicit affect measure (number of negative memories for events before age 14), but not with an explicit mood scale, and in both of their studies AI moderated the effect of stress on negative mood (Study 1) and daily hassles (Study 2), but was not per se associated with it.

AI also has virtually no variance overlap with trait measures of emotionality and temperament. In a large-scale study of German students, Schultheiss and Brunstein (2001) failed to observe significant overlap between AI and extraversion, neuroticism and other Big Five traits. Likewise, in a large sample of U.S. students Pang and Schultheiss (2005) found correlations close to zero between $\mathrm{AI}$ and anxiety and impulsivity as assessed with Carver and White's (1994) BIS/BAS scales, which have been found to predict hemispheric differences in prefrontal activation in previous research (Sutton \& Davidson, 1997). These findings suggest that the AI measure has little overlap with explicit measures of emotionality, which have been robustly linked to hemispheric differences in prefrontal activation (see, Davidson, 2000), but reflects a latent disposition to regulate hemispheric functions in the presence of stress or challenges and may thus predict variance in functional hemispheric asymmetries above and beyond explicit measures. Thus, a person with high levels of trait anxiety may chronically have slightly more dominant RH functions, regardless of her or his level of AI. But whether this imbalance between $\mathrm{LH}$ and $\mathrm{RH}$ functioning becomes more severe under stress, remains the same, or even reverses in favor of LH functions, will depend on whether the person has high, medium, or low AI levels, respectively.

\section{Limitations and Future Directions}

While our findings are consistent with the AI-laterality hypothesis, we acknowledge that performance measures of laterality like the DPT represent indirect measures of brain function and are less precise than anatomical measures (e.g., functional magnetic resonance imaging). Moreover, neither our review of the AI and brain asymmetry literature, nor the results of our present research allow us to specify in any detail which brain functions are directly 
involved in the AI-laterality effect. Clearly, we would not, on the basis of our present findings, claim that all brain functions are more lateralized in stressed high-AI individuals. The finding that AI is related to lateralized attentional processes, but not to lateralization of motor functions (Study 2), already speaks to this point. Thus, future work needs to carefully delineate which specific lateralized cognitive, emotional, and physiological functions are enhanced and which, if any, are less activated due to variations in $\mathrm{AI}$ in stressful situations. In addition, research using brain imaging approaches or comparisons between patient populations with lateralized brain lesions and nonlesioned control participants would be helpful to pinpoint specific neurobiological substrates or networks involved in the AI-laterality effect.

Consistent with our evaluation of the measurement credentials in the introduction, we found the AI measure to have only low to moderate internal consistency. While this issue could be relatively easily remedied by increasing test length, we suggest that future research should also explore whether reliability — and validity — of the AI measure can be increased by extending it to other forms of verbal negation (e.g., no, none, never) or, more generally, to other, correlated linguistic markers. For instance, in analyses of various text sources, Pennebaker and King (1999) found that negations loaded on one factor with exclusive words (e.g., but, without, except), tentative words (e.g., perhaps, maybe), and the absence of inclusion words (e.g., and, with). Although the authors did not report an internal consistency estimate for the composite of all scales represented by this factor, it is almost certainly substantially higher than the alphas of .47 to .61 they report for each of the word categories making up the factor. If that is the case, it would be particularly interesting to find out whether the overall scale including negations is a better, equal, or worse predictor of lateralized hemispheric function under stress than the frequency of negations (i.e., AI) by itself.

In addition, we think it worthwhile to examine the discriminant and convergent validity of AI with other measures of emotion regulation and coping, such as individual differences in reappraisal and emotion suppression (e.g., John \& Gross, 2004), ego resiliency (e.g., Block \& Kremen, 1996), or action and state orientation (e.g., Kuhl, 1981). Conceptually, AI is similar to these measures in that it predicts individual differences in the magnitude and quality of people's emotional responses to the same stressors and challenges. McClelland (1979; see also Langens \& Stucke, 2005) made this point most clearly when he portrayed AI as a general tendency to inhibit the expression of emotional-motivational impulses. However, AI is different from these measures in that it appears to represent a latent, automatic tendency to recruit lateralized brain functions for coping with a stressor which does not appear to be captured well by questionnaire measures of emotional traits and states. ${ }^{6}$ We therefore expect the shared variance between AI and self-report measures of emotion regulation and coping to be relatively small. Thus, AI may contribute to our knowledge about and understanding of emotion regulation processes above and beyond established measures.

Finally, one particularly intriguing aspect of AI that it shares with other content coding measures of verbal material is that it can be used with any type of verbal material and thus allows researchers and clinicians to study an important aspect of psychological functioning in individuals who are not available for direct assessment (e.g., politicians, deceased historical figures, or patients who are unwilling or unable to undergo traditional forms of psychological assessment). For instance, AI has been scored in U.S. presidents' inaugural speeches (e.g., Winter, 1991, 2001), letters of CEOs to the stockholders of their companies (Diaz, 1982, in McClelland, 1987), and, with a slightly extended scoring criterion, in the diaries of substance abuse patients and the journal abstracts of social scientists (Pennebaker \& King, 1999). Thus, the verbal material that people generate in their private and professional lives may provide clues to how they deal with stressful challenges; whether they are more likely to recruit $\mathrm{RH}$ functions, $\mathrm{LH}$ functions, or both in equal measure. The more we learn about the construct validity of AI, the better we will be able to read between the lines (or, as it were, the "nots") of individuals' verbal productions and understand their typical style of dealing with stress. We therefore hope that the findings reported in this paper will help make the AI measure worthy of further research.

\footnotetext{
${ }^{6}$ Although state-of-the-art accounts of emotion regulation, such as Gross's (1998) influential model, emphasize the emotion regulation can also occur automatically and unconsciously-a view that would fit the AI construct-so far most researchers rely on self-report measures to assess emotion regulation.
}

\section{References}

Adolphs, R. (2002). Recognizing emotion from facial expressions: Psychological and neurological mechanisms. Behavioral and Cognitive Neuroscience Reviews, 1, 21-61.

Aron, A. R., Robbins, T. W., \& Poldrack, R. A. (2004). Inhibition and the right inferior frontal cortex. Trends in Cognitive Sciences, 8, 170-177.

Beringer, J. (2000). Experimental Run Time System (ERTS) (Version 3.32) [computer software]. Frankfurt, Germany: BeriSoft Cooperation.

Block, J., \& Kremen, A. M. (1996). IQ and ego-resiliency: Conceptual and empirical connections and separateness. Journal of Personality and Social Psychology, 70, 349-361.

Borod, J. C. (1992). Interhemispheric and intrahemispheric control of emotion: A focus on unilateral brain damage. Journal of Consulting and Clinical Psychology, 60, 339-348.

Buck, R., \& Duffy, R. J. (1980). Nonverbal communication of affect in brain-damaged patients. Cortex, 16, 351-362.

Carver, C. S., \& White, T. L. (1994). Behavioral inhibition, behavioral activation, and the affective responses to impending reward and punishment: The BIS/BAS scales. Journal of Personality and Social Psychology, 67, 319-333.

Cohen, J., Cohen, P., West, S. G., \& Aiken, L. S. (2003). Applied multiple regression/correlation analysis for the behavioral sciences (3rd ed.). Mahwah, NJ: Erlbaum.

Davidson, R. J. (2000). Affective style, psychopathology, and resilience: Brain mechanisms and plasticity. American Psychologist, 55, 11961214.

Davidson, R. J., \& Hugdahl, K. (Eds.). (1995). Brain asymmetry. Cambridge, MA: MIT Press.

Davidson, R. J., Kabat-Zinn, J., Schumacher, J., Rosenkranz, M., Muller, D., Santorelli, S. F., et al. (2003). Alterations in brain and immune function produced by mindfulness meditation. Psychosomatic Medicine, 65, 564-570.

Dimberg, U., \& Petterson, M. (2000). Facial reactions to happy and angry facial expressions: Evidence for right hemisphere dominance. Psychophysiology, 37, 693-696.

Ellis, R. J., \& Oscar-Berman, M. (1989). Alcoholism, aging, and functional cerebral asymmetries. Psychological Bulletin, 106, 128-147. 
Evert, D. L., \& Oscar-Berman, M. (2001). Selective attentional processing and the right hemisphere: Effects of aging and alcoholism. Neuropsychology, 15, 452-461.

Fontana, A. F., Rosenberg, R. L., Marcus, J. L., \& Kerns, R. D. (1987). Type A behavior pattern, inhibited power motivation, and activity inhibition. Journal of Personality and Social Psychology, 52, 177-183.

Goldberg, E. (2001). The executive brain: Frontal lobes and the civilized mind. New York: Oxford University Press.

Gray, J. A. (1987). The psychology of fear and stress. London: Cambridge University Press.

Gross, J. J. (1998). The emerging field of emotion regulation: An integrative review. Review of General Psychology, 2, 271-299.

Heilman, K. M. (1995). Attentional asymmetries. In R. J. Davidson \& K. Hugdahl (Eds.), Brain asymmetry (pp. 217-234). Cambridge, MA: MIT Press.

Heller, W., Koven, N. S., \& Miller, G. A. (2003). Regional brain activity in anxiety and depression, cognition/emotion interaction, and emotion regulation. In K. Hugdahl \& R. J. Davidson (Eds.), The asymmetrical brain (pp. 533-564). Cambridge, MA: MIT Press.

Hellige, J. B., \& Sergent, J. (1986). Role of task factors in visual field asymmetries. Brain and Cognition, 5, 200-222.

Hilz, M. J., Dutsch, M., Perrine, K., Nelson, P. K., Rauhut, U., \& Devinsky, O. (2001). Hemispheric influence on autonomic modulation and baroreflex sensitivity. Annals of Neurology, 49, 575-584.

Hugdahl, K. (1995). Classical conditioning and implicit learning: The right hemisphere hypothesis. In R. J. Davidson \& K. Hugdahl (Eds.), Brain asymmetry (pp. 235-268). Cambridge, MA: MIT Press.

Hugdahl, K., \& Davidson, R. J. (Eds.). (2003). The asymmetrical brain. Cambridge, MA: MIT Press.

Hutner, N., \& Oscar-Berman, M. (1996). Visual laterality patterns for the perception of emotional words in alcoholic and aging individuals. Journal of Studies on Alcohol, 57, 144-154.

John, O. P., \& Gross, J. J. (2004). Healthy and unhealthy emotion regulation: Personality processes, individual differences, and life span development. Journal of Personality, 72, 1301-1334.

Kalin, R., McClelland, D. C., \& Kahn, M. (1965). The effects of male social drinking. Journal of Personality and Social Psychology, 1, 441452.

Keller, J., Nitschke, J. B., Bhargava, T., Deldin, P. J., Gergen, J. A., Miller, G. A., et al. (2000). Neuropsychological differentiation of depression and anxiety. Journal of Abnormal Psychology, 109, 3-10.

Kuhl, J. (1981). Motivational and functional helplessness: The moderating effect of state versus action orientation. Journal of Personality and Social Psychology, 40, 155-170.

Langens, T. A., \& Stucke, T. S. (2005). Stress and mood: The moderating role of activity inhibition. Journal of Personality, 73(1), 47-78.

Mason, A., \& Blankenship, V. (1987). Power and affiliation motivation, stress, and abuse in intimate relationships. Journal of Personality and Social Psychology, 52, 203-210.

Matsumoto, D., \& Ekman, P. (1988). Japanese and Caucasian facial expressions of emotion (JACFEE) and neutral faces (JACNeuF) [Slides]. San Francisco: Author.

Matthews, G., Jones, D. M., \& Chamberlain, A. G. (1990). Refining the measurement of mood: The UWIST Mood Adjective Checklist. British Journal of Psychology, 81, 17-42.

Maxwell, J. S., Shackman, A. J., \& Davidson, R. J. (2005). Unattended facial expressions asymmetrically bias the concurrent processing of nonemotional information. Journal of Cognitive Neuroscience, 17, $1386-1395$.

McClelland, D. C. (1979). Inhibited power motivation and high blood pressure in men. Journal of Abnormal Psychology, 88, 182-190.

McClelland, D. C. (1987). Human motivation. New York: Cambridge University Press.
McClelland, D. C., \& Boyatzis, R. E. (1982). Leadership motive pattern and long-term success in management. Journal of Applied Psychology, 67, 737-743.

McClelland, D. C., Davidson, R. J., \& Saron, C. (1985). Stressed power motivation, sympathetic activation, immune function, and illness. Advances, 2, 42-52.

McClelland, D. C., Davis, W. N., Kalin, R., \& Wanner, E. (1972). The drinking man. New York: Free Press.

McClelland, D. C., Floor, E., Davidson, R. J., \& Saron, C. (1980). Stressed power motivation, sympathetic activation, immune function, and illness. Journal of Human Stress, 6, 11-19.

McClelland, D. C., \& Franz, C. E. (1992). Motivational and other sources of work accomplishments in mid-life: A longitudinal study. Journal of Personality, 60, 679-707.

McClelland, D. C., \& Jemmott, J. B. (1980). Power motivation, stress and physical illness. Journal of Human Stress, 6, 6-15.

McClelland, D. C., \& Kirshnit, C. (1988). The effect of motivational arousal through films on salivary immunoglobulin A. Psychology and Health, 2, 31-52.

Meador, K. J., Loring, D. W., Ray, P. G., Helman, S. W., Vazquez, B. R., \& Neveu, P. J. (2004). Role of cerebral lateralization in control of immune processes in humans. Annals of Neurology, 55, 840-844.

Mogg, K., \& Bradley, B. P. (1999). Some methodological issues in assessing attentional biases for threatening faces in anxiety: A replication study using a modified version of the probe detection task. Behaviour Research and Therapy, 37, 595-604.

Neveu, P. J. (2002). Cerebral lateralization and the immune system. International Review of Neurobiology, 52, 303-323.

Pang, J. S., \& Schultheiss, O. C. (2005). Assessing implicit motives in U.S. college students: Effects of picture type and position, gender and ethnicity, and cross-cultural comparisons. Journal of Personality Assessment, 85, 280-294.

Patterson, C. M., \& Newman, J. P. (1993). Reflectivity and learning from aversive events: Toward a psychological mechanism for the syndromes of disinhibition. Psychology Review, 100, 716-736.

Pennebaker, J. W., Francis, M. E., \& Booth, R. J. (2001). Linguistic Inquiry and Word Count (LIWC): LIWC2001. Mahwah, NJ: Erlbaum.

Pennebaker, J. W., \& King, L. A. (1999). Linguistic styles: Language use as an individual difference. Journal of Personality and Social Psychology, 77, 1296-1312.

Posner, M. I. (1995). Attention in cognitive neuroscience: An overview. In M. S. Gazzaniga (Ed.), The cognitive neurosciences (pp. 615-624). Cambridge, MA: Bradford/MIT Press.

Reuter-Lorenz, P., \& Davidson, R. J. (1981). Differential contributions of the two cerebral hemispheres to the perception of happy and sad faces. Neuropsychologia, 19, 609-613.

Sackeim, H. A., Gur, R. C., \& Saucy, M. C. (1978). Emotions are expressed more intensely on the left side of the face. Science, 202(4366), 434-436.

Sapolsky, R. M. (1992). Neuroendocrinology of the stress-response. In J. B. Becker, S. M. Breedlove, \& D. Crews (Eds.), Behavioral endocrinology (pp. 287-324). Cambridge MA: MIT Press.

Saron, C. D., Foxe, J. J., Schroeder, C. E., \& Vaughan, H. G. (2003). Complexities of interhemispheric communication in sensorimotor tasks revealed by high-density event-related potential mapping. In K. Hugdahl \& R. J. Davidson (Eds.), The asymmetrical brain (pp. 341-408). Cambridge, MA: MIT Press.

Schultheiss, O. C., \& Brunstein, J. C. (2001). Assessing implicit motives with a research version of the TAT: Picture profiles, gender differences, and relations to other personality measures. Journal of Personality Assessment, 77, 71-86.

Schultheiss, O. C., \& Brunstein, J. C. (2002). Inhibited power motivation and persuasive communication: A lens model analysis. Journal of Personality, $70,553-582$. 
Schultheiss, O. C., \& Hale, J. A. (2007). Implicit motives modulate attentional orienting to perceived facial expressions of emotion. Motivation and Emotion, 31, 13-24.

Schultheiss, O. C., \& Pang, J. S. (2007). Measuring implicit motives. In R. W. Robins, R. C. Fraley, \& R. Krueger (Eds.), Handbook of research methods in personality psychology (pp. 322-344). New York: Guilford Press.

Schultheiss, O. C., \& Rohde, W. (2002). Implicit power motivation predicts men's testosterone changes and implicit learning in a contest situation. Hormones and Behavior, 41, 195-202.

Schultheiss, O. C., Wirth, M. M., Torges, C. M., Pang, J. S., Villacorta, M. A., \& Welsh, K. M. (2005). Effects of implicit power motivation on men's and women's implicit learning and testosterone changes after social victory or defeat. Journal of Personality and Social Psychology, 88, 174-188.

Sutton, S. K., \& Davidson, R. J. (1997). Prefrontal brain asymmetry: A biological substrate of the behavioral approach and inhibition systems. Psychological Science, 8, 204-210.

Toga, A. W., \& Thompson, P. M. (2003). Mapping brain asymmetry. Nature Reviews: Neuroscience, 4, 37-48.

Winter, D. G. (1991). Measuring personality at a distance: Development of an integrated system for scoring motives in running text. In D. J. Ozer,
J. M. Healy, \& A. J. Stewart (Eds.), Perspectives in personality (Vol. 3, pp. 59-89). London: Jessica Kingsley.

Winter, D. G. (2001). Measuring Bush's motives. ISPP News: International Society of Political Psychology, 12, 9.

Wirth, M. M., Welsh, K. M., \& Schultheiss, O. C. (2006). Salivary cortisol changes in humans after winning or losing a dominance contest depend on implicit power motivation. Hormones and Behavior, 49, 346-352.

Wittling, W. (1990). Psychophysiological correlates of human brain asymmetry: Blood pressure changes during lateralized presentation of an emotionally laden film. Neuropsychologia, 28, 457-470.

Wittling, W. (1995). Brain asymmetry in the control of autonomic-physiologic activity. In R. J. Davidson \& K. Hugdahl (Eds.), Brain asymmetry (pp. 305-357). Cambridge, MA: MIT Press.

Wittling, W. (1997). Brain asymmetry and autonomic control of the heart. European Psychologist, 2, 313-327.

Wittling, W., Block, A., Genzel, S., \& Schweiger, E. (1998). Hemisphere asymmetry in parasympathetic control of the heart. Neuropsychologia, 36, 461-468.

Received October 23, 2007

Revision received September 22, 2008

Accepted October 8, 2008

\section{E-Mail Notification of Your Latest Issue Online!}

Would you like to know when the next issue of your favorite APA journal will be available online? This service is now available to you. Sign up at http://notify.apa.org/ and you will be notified by e-mail when issues of interest to you become available! 